\title{
Ophthalmologica
}

\section{Elfriede-Aulhorn-Preis der Deutschen Ophthalmologischen Gesellschaft}

Der 'Elfriede-Aulhorn-Preis' ist von der Neuro-ophthalmologischen Gesellschaft - Mehr forschen - besser sehen - e.V. gestiftet worden, um in Erinnerung an die Namensträgerin des Preises die Forschung im Bereich der Physiologie und Pathophysiologie des Sehens sowie der Neuro-Ophthalmologie zu fördern. Der Preis wird für besonders wertvolle Arbeiten in den genannten Wissenschaftsgebieten verliehen, insbesondere für die Entwicklung von neuen, für Klinik und augenärztliche Praxis wichtigen sinnesphysiologischen Untersuchungsmethoden oder Studien zur Verbesserung diagnostischer oder therapeutischer Verfahren bei neuro-ophthalmologischen Erkrankungen.

Der mit EURO 4000.- dotierte Preis wird im zweijährigen Turnus verliehen. Er kann bei entsprechendem Beschluss der Kommission auch unter mehreren Bewerbern aufgeteilt werden.

- Bewerber sollten im deutschen Sprachraum arbeiten.

- Die eingereichte Arbeit muss bereits in deutscher oder englischer Sprache veröffentlicht oder zur Veröffentlichung angenommen worden sein.

- Eine von der Deutschen Ophthalmologischen Gesellschaft (DOG) eingerichtete Kommission trifft die Vergabeentscheidung. Die Preisverleihung erfolgt im Rahmen der Jahrestagung der DOG.

Bewerbungen sind bis zum 15. Mai 2006 an den Federführenden der Kommission zu richten:

Herrn Prof. Dr. med. Eberhart Zrenner

Universitäts-Augenklinik Tübingen

Schleichstrasse 12-16, DE-72076 Tübingen

Fax +49 807071/29 5038.

Es wird gebeten, einem kurzen Anschreiben folgende Unterlagen in siebenfacher Ausfertigung beizufügen:

- einen Sonderdruck der preiswürdig erachteten Arbeit oder bei sehr umfangreichen Arbeiten eine Zusammenfassung von ca. 10 Seiten,

- ein Verzeichnis der publizierten Arbeiten,

- eine tabellarische Darstellung des beruflichen Werdegangs. 